On uniqueness of Lamé coefficients from partial Cauchy data in three dimensions

This article has been downloaded from IOPscience. Please scroll down to see the full text article.

2012 Inverse Problems 28125002

(http://iopscience.iop.org/0266-5611/28/12/125002)

View the table of contents for this issue, or go to the journal homepage for more

Download details:

IP Address: 205.175.121.224

The article was downloaded on 21/12/2012 at 22:01

Please note that terms and conditions apply. 


\title{
On uniqueness of Lamé coefficients from partial Cauchy data in three dimensions
}

\author{
Oleg Yu Imanuvilov ${ }^{1}$, Gunther Uhlmann ${ }^{2,3}$ and Masahiro Yamamoto ${ }^{4}$ \\ ${ }^{1}$ Department of Mathematics, Colorado State University, 101 Weber Building, Fort Collins, \\ CO 80523, USA \\ ${ }^{2}$ Department of Mathematics, UC Irvine, Irvine, CA 92697, USA \\ ${ }^{3}$ Department of Mathematics, University of Washington, Seattle, WA 98195, USA \\ ${ }^{4}$ Department of Mathematical Sciences, The University of Tokyo, Komaba, Meguro, Tokyo 153, \\ Japan \\ E-mail: oleg@math.colostate.edu,gunther@math.washington.edu and myama@ms.u-tokyo.ac.jp
}

Received 20 May 2012, in final form 1 October 2012

Published 6 November 2012

Online at stacks.iop.org/IP/28/125002

\begin{abstract}
For the Lamé system, we prove in the three-dimensional case that both Lamé coefficients are uniquely recovered from partial Cauchy data on an arbitrary open subset of the boundary provided that the coefficient $\mu$ is a constant.
\end{abstract}

In a bounded domain $\Omega \subset \mathbf{R}^{3}$ with smooth boundary, we consider the Lamé system

$$
\sum_{j, k, l=1}^{3} \frac{\partial}{\partial x_{j}}\left(C_{i j k l} \frac{\partial u^{k}}{\partial x_{l}}\right)=0 \quad \text { in } \Omega, 1 \leqslant i \leqslant 3,
$$

and

$$
\left.u\right|_{\partial \Omega}=f
$$

where

$$
C_{i j k l}=\lambda(x) \delta_{i j} \delta_{k l}+\mu(x)\left(\delta_{i k} \delta_{j l}+\delta_{i l} \delta_{j k}\right), \quad 1 \leqslant i, j, k, l \leqslant 3
$$

with the Kronecker delta $\delta_{i j}$. The functions $\lambda$ and $\mu$ are called the Lamé coefficients, and $u(x)=\left(u^{1}(x), u^{2}(x), u^{3}(x)\right)$ is the displacement. Assume that

$$
\mu(x)>0 \quad \text { on } \bar{\Omega}, \quad(3 \lambda+2 \mu)(x)>0 \quad \text { on } \bar{\Omega} .
$$

We set

$$
\Lambda_{\lambda, \mu} f=\left(\sum_{j, k, l=1}^{3} v_{j} C_{1 j k l} \frac{\partial u^{k}}{\partial x_{l}}, \sum_{j, k, l=1}^{3} v_{j} C_{2 j k l} \frac{\partial u^{k}}{\partial x_{l}}, \sum_{j, k, l=1}^{3} v_{j} C_{3 j k l} \frac{\partial u^{k}}{\partial x_{l}}\right)
$$

where $v=\left(v_{1}, v_{2}, v_{3}\right)$ is the outward unit normal vector to $\partial \Omega$ and $u$ is the solution to (1) and (2). Denote

$\mathcal{L}_{\lambda, \mu}(x, D) u=\left(\sum_{j, k, l=1}^{3} \frac{\partial}{\partial x_{j}}\left(C_{1 j k l} \frac{\partial u^{k}}{\partial x_{l}}\right), \sum_{j, k, l=1}^{3} \frac{\partial}{\partial x_{j}}\left(C_{2 j k l} \frac{\partial u^{k}}{\partial x_{l}}\right), \sum_{j, k, l=1}^{3} \frac{\partial}{\partial x_{j}}\left(C_{3 j k l} \frac{\partial u^{k}}{\partial x_{l}}\right)\right)$. 
The partial Cauchy data $\mathcal{C}_{\lambda, \mu}$ are defined by

$\mathcal{C}_{\lambda, \mu}=\left\{\left.\left(u, \Lambda_{\lambda, \mu} u\right)\right|_{\tilde{\Gamma}} ; \mathcal{L}_{\lambda, \mu}(x, D) u=0 \quad\right.$ in $\left.\Omega,\left.u\right|_{\partial \Omega}=f, \quad \operatorname{supp} f \subset \widetilde{\Gamma}, f \in H^{\frac{3}{2}}(\partial \Omega)\right\}$.

Here, $\widetilde{\Gamma}$ is an arbitrarily fixed open subset of $\partial \Omega$. We set $\Gamma_{0}=\partial \Omega \backslash \widetilde{\Gamma}$.

In this paper, we consider the following inverse problem. Suppose that the partial Cauchy data $\mathcal{C}_{\lambda, \mu}$ are given. Can we determine the Lamé coefficients $\lambda$ and $\mu$ ?

This inverse problem has been studied since the 1990s. A linearized version of this inverse problem for full data was studied by Ikehata [7]. In two dimensions, Akamatsu et al [1] proved that for the case of full Cauchy data $(\widetilde{\Gamma}=\partial \Omega)$ one can recover the Lamé coefficients and their normal derivatives of arbitrary orders on the boundary provided that the Lamé coefficients are $C^{\infty}$ functions. This boundary determination result was extended by Nakamura and Uhlmann [17] to higher dimensions. In [15], Nakamura and Uhlmann for the case of full Cauchy data established that in two dimensions the Lamé coefficients are uniquely determined, assuming that they are sufficiently close to a pair of positive constants. Recently, Imanuvilov and Yamamoto in [13] proved for the two-dimensional case that the Lamé coefficient $\lambda$ can be recovered from partial Cauchy data if the coefficient $\mu$ is some positive constant. In two dimensions, there is another possible way to prove a local uniqueness result for $\lambda$ and $\mu$ which are close to constants: the proof is a combination of [1] and a local uniqueness result for the Love-Kirchhoff plate equation in [8], and the equivalence of the two problems is proved in [9].

For the three-dimensional case, the uniqueness for both Lamé coefficients is proved provided that $\mu$ is close to a positive constant $[6,16]$ and the proof relies on construction of complex geometric optics solution $[5,18]$. Our result relies on these uniqueness results for the case of full data.

All the above works are concerned with the full Cauchy data (i.e. $\widetilde{\Gamma}=\partial \Omega$ ). The recovery of Lamé coefficients by partial Cauchy data on an arbitrary sub-boundary is useful from the practical point of view, because one can limit input and measurement subsets of $\partial \Omega$ as much as possible. In the case of partial Cauchy data for the Lamé system, unlike the case of the Schrödinger operator, the construction of complex geometric optics solutions seems to be possible only for a dense set of Lamé coefficients. To the best of our knowledge, there are no results on the unique recovery of the Lamé coefficients from the partial Cauchy data in the three-dimensional case. The purpose of this paper is to prove such uniqueness in three dimensions.

Finally, we mention that this inverse problem is closely related to the method known as electrical impedance tomography (EIT). EIT is used in prospection of oil and minerals and in medical imaging in detecting breast cancer, pulmonary edema, etc. For the mathematical treatments of this problem, we refer, e.g., to [2-4, 11, 12, 14, 19] and the review paper [20].

Our result is the following theorem.

Theorem 0.1. Let $\mu_{1}, \mu_{2}$ be some positive constants and $\lambda_{1}, \lambda_{2} \in C^{\infty}(\bar{\Omega})$ be some functions satisfying (3) and $\lambda_{1}=\lambda_{2}$ on $\Gamma_{0}$. If $\mathcal{C}_{\lambda_{1}, \mu_{1}}=\mathcal{C}_{\lambda_{2}, \mu_{2}}$, then $\left(\lambda_{1}, \mu_{1}\right)=\left(\lambda_{2}, \mu_{2}\right)$.

Proof. The proof consists in showing that from partial Cauchy data one can recover the full data. First, following [17], we prove that

$$
\left(\lambda_{1}, \mu_{1}\right)=\left(\lambda_{2}, \mu_{2}\right) \quad \text { on } \widetilde{\Gamma} \text { and } \frac{\partial \lambda_{1}}{\partial v}=\frac{\partial \lambda_{2}}{\partial v} \text { on } \widetilde{\Gamma} .
$$

Let $u_{j} \in H^{2}(\Omega), j=1,2$, be functions such that

$$
\mathcal{L}_{\lambda_{j}, \mu_{j}}(x, D) u_{j}=0 \quad \text { in } \Omega,\left.\quad u_{j}\right|_{\partial \Omega}=f,
$$


where supp $f \subset \widetilde{\Gamma}$. Since the partial Cauchy data are the same, we obtain

$$
\Lambda_{\lambda_{1}, \mu_{1}} u_{1}=\Lambda_{\lambda_{2}, \mu_{2}} u_{2} \text { on } \widetilde{\Gamma},
$$

where $\Lambda_{\lambda_{i}, \mu_{i}}, i=1,2$, are defined in (4).

Assume for the moment that $u_{1} \in C^{2+\alpha}(\bar{\Omega})$ for some $\alpha$ from $(0,1)$. The regularity results for the Lamé system imply immediately that $u_{2} \in C^{2+\alpha}(\bar{\Omega})$.

By (5) and $3 \lambda_{j}+2 \mu_{j}>0$ on $\bar{\Omega}, j=1,2$, we can prove

$$
\left(u_{1}, \frac{\partial u_{1}}{\partial v}\right)=\left(u_{2}, \frac{\partial u_{2}}{\partial v}\right) \text { on } \widetilde{\Gamma} \text {. }
$$

Moreover, from (5), (8) and equation (6), we conclude

$$
\left(u_{1}, \frac{\partial u_{1}}{\partial v}, \frac{\partial^{2} u_{1}}{\partial x_{i} \partial x_{k}}\right)=\left(u_{2}, \frac{\partial u_{2}}{\partial v}, \frac{\partial^{2} u_{2}}{\partial x_{i} \partial x_{k}}\right) \quad \text { on } \quad \widetilde{\Gamma}, \quad \forall i, k \in\{1,2,3\}
$$

Hence,

$$
\left(\operatorname{rot} u_{1}, \frac{\partial \operatorname{rot} u_{1}}{\partial v}\right)=\left(\operatorname{rot} u_{2}, \frac{\partial \operatorname{rot} u_{2}}{\partial v}\right) \quad \text { on } \widetilde{\Gamma} \text {. }
$$

As for the proof of (9) and (8), we refer, for instance, to [10].

Since the functions $\mu_{j}$ are assumed to be constants, from (5) we conclude that

$$
\mu:=\mu_{1}=\mu_{2} \quad \text { in } \Omega \text {. }
$$

For the constant $\mu$, we note that

$$
\mathcal{L}_{\lambda, \mu}(x, D) u=\mu \Delta u+(\mu+\lambda) \nabla \operatorname{div} u+(\operatorname{div} u) \nabla \lambda .
$$

Applying to equation (6) the operator rot and using the fact that $\mu_{j}$ is constant, we obtain

$$
\mu_{j} \Delta \operatorname{rot} u_{j}=0 \quad \text { in } \Omega, j=1,2 .
$$

Equality (10) and the uniqueness of the solution for the Cauchy problem for the Laplace equation imply

$$
\operatorname{rot} u_{1}=\operatorname{rot} u_{2} \quad \text { in } \Omega \text {. }
$$

The Lamé operator, with the coefficient $\mu=$ const, can be written in the form $\mathcal{L}(x, D) u=$ $\nabla((\lambda+2 \mu) \operatorname{div} u)-\mu \operatorname{rot} \operatorname{rot} u$. Then, using (11) and (13), we obtain

$$
\nabla\left(\left(\lambda_{1}+2 \mu\right) \operatorname{div} u_{1}\right)=\nabla\left(\left(\lambda_{2}+2 \mu\right) \operatorname{div} u_{2}\right) \quad \text { in } \Omega .
$$

Hence, $\left(\lambda_{1}+2 \mu\right) \operatorname{div} u_{1}-\left(\lambda_{2}+2 \mu\right) \operatorname{div} u_{2}$ is a constant function in $\Omega$. Since $\left(\lambda_{1}+2 \mu\right) \operatorname{div} u_{1}=$ $\left(\lambda_{2}+2 \mu\right) \operatorname{div} u_{2}$ on $\widetilde{\Gamma}$ by (5) and (8), equation (14) implies

$$
\left(\lambda_{1}+2 \mu\right) \operatorname{div} u_{1}=\left(\lambda_{2}+2 \mu\right) \operatorname{div} u_{2} \quad \text { in } \Omega .
$$

From (11), (15), (13) and the assumption $\left.\left(\lambda_{1}-\lambda_{2}\right)\right|_{\Gamma_{0}}=0$, we conclude

$$
\frac{\partial u_{1}}{\partial v}=\frac{\partial u_{2}}{\partial v} \quad \text { on } \Gamma_{0}
$$

Indeed in order to obtain equality (16) observe that rotations preserve the isotropicity of the Lamé system. Therefore, after choosing an arbitrary point $x \in \Gamma_{0}$, we rotate the coordinates to have $v(x)=(0,0,1)$. Then, we obtain

$$
\frac{\partial u_{1, j}}{\partial x_{k}}(x)=\frac{\partial u_{2, j}}{\partial x_{k}}(x), \quad \forall j \in\{1,2,3\}, \quad \forall k \in\{1,2\} .
$$

Hence, equality (13) implies that $\frac{\partial u_{1, j}}{\partial v}(x)=\frac{\partial u_{2, j}}{\partial v}(x)$ for $j=1,2$ and (15) implies that $\frac{\partial u_{1,3}}{\partial v}(x)=\frac{\partial u_{2,3}}{\partial v}(x)$. 
By (16), if $f \in C^{2+\alpha}(\partial \Omega), \operatorname{supp} f \subset \widetilde{\Gamma}$ in (5), then $\frac{\partial u_{1}}{\partial v}=\frac{\partial u_{2}}{\partial v}$ on $\partial \Omega$. By a density argument, the above relation holds for the slightly relaxed regularity assumption on the function $f$. Namely, the function $f$ belongs to $H^{\frac{3}{2}}(\partial \Omega)$. Hence, we removed the assumption that $u_{1} \in C^{2+\alpha}(\bar{\Omega})$.

Next, let $f \in H^{\frac{3}{2}}(\partial \Omega)$ and the functions $v_{j} \in H^{2}(\Omega)$ be the solutions of the following boundary value problems:

$$
\mathcal{L}_{\lambda_{j}, \mu_{j}}(x, D) v_{j}=0 \quad \text { in } \Omega,\left.\quad v_{j}\right|_{\partial \Omega}=f, \quad j \in\{1,2\} .
$$

We claim that

$$
\frac{\partial v_{1}}{\partial v}=\frac{\partial v_{2}}{\partial v} \quad \text { on } \quad \widetilde{\Gamma} .
$$

Indeed, let $w_{j} \in H^{2}(\Omega)$ be a solution to the Lamé system

$$
\mathcal{L}_{\lambda_{j}, \mu_{j}}(x, D) w_{j}=0 \quad \text { in } \quad \Omega,\left.\quad w_{j}\right|_{\partial \Omega}=g, \quad j \in\{1,2\},
$$

where $g \in H^{\frac{3}{2}}(\partial \Omega)$ and supp $g \subset \widetilde{\Gamma}$ is an arbitrary function. Taking the scalar product of equation (17) with $w_{j}$ and integrating by parts, we have

$$
\begin{aligned}
0= & \int_{\Omega}\left(\mathcal{L}_{\lambda_{j}, \mu_{j}}(x, D) v_{j}, w_{j}\right) \mathrm{d} x=\int_{\Omega}\left(v_{j}, \mathcal{L}_{\lambda_{j}, \mu_{j}}(x, D) w_{j}\right) \mathrm{d} x \\
& +\int_{\partial \Omega}\left(\left(\Lambda_{\lambda_{j}, \mu_{j}} v_{j}, w_{j}\right)-\left(\Lambda_{\lambda_{j}, \mu_{j}} w_{j}, v_{j}\right)\right) \mathrm{d} \sigma \\
= & \int_{\partial \Omega}\left(\left(\Lambda_{\lambda_{j}, \mu_{j}} v_{j}, g\right)-\left(\Lambda_{\lambda_{j}, \mu_{j}} w_{j}, f\right)\right) \mathrm{d} \sigma=\int_{\widetilde{\Gamma}}\left(\Lambda_{\lambda_{j}, \mu_{j}} v_{j}, g\right) \mathrm{d} \sigma-\int_{\partial \Omega}\left(\Lambda_{\lambda_{j}, \mu_{j}} w_{j}, f\right) \mathrm{d} \sigma \\
= & \int_{\widetilde{\Gamma}}\left(\Lambda_{\lambda_{j}, \mu_{j}} v_{j}, g\right) \mathrm{d} \sigma-\int_{\partial \Omega}\left(\Lambda_{\lambda_{1}, \mu_{1}} w_{1}, f\right) \mathrm{d} \sigma,
\end{aligned}
$$

where $\mathrm{d} \sigma$ denotes the surface measure.

This integral identity implies

$$
\Lambda_{\lambda_{1}, \mu_{1}} v_{1}=\Lambda_{\lambda_{2}, \mu_{2}} v_{2} \quad \text { on } \quad \widetilde{\Gamma} .
$$

Repeating the arguments in (12)-(16), we conclude

$$
\frac{\partial v_{1}}{\partial v}=\frac{\partial v_{2}}{\partial v} \quad \text { on } \Gamma_{0}
$$

Hence, by (16) and (20), the following full Cauchy data are equal:

$$
\widetilde{\mathcal{C}}_{\lambda_{1}, \mu_{1}}=\widetilde{\mathcal{C}}_{\lambda_{2}, \mu_{2}}
$$

where

$$
\widetilde{\mathcal{C}}_{\lambda, \mu}=\left\{\left.\left(u, \Lambda_{\lambda, \mu} u\right)\right|_{\partial \Omega} ; \mathcal{L}_{\lambda, \mu}(x, D) u=0 \quad \text { in } \Omega,\left.u\right|_{\partial \Omega}=f, f \in H^{\frac{3}{2}}(\partial \Omega)\right\} .
$$

Applying the result of [6], we obtain that $\lambda_{1}=\lambda_{2}$.

\section{Acknowledgments}

The authors thank the anonymous referees and board member for invaluable comments. The first author was partly supported by GCOE program of The University of Tokyo. The second author was partly supported by the NSF. 


\section{References}

[1] Akamatsu M, Nakamura G and Steinberg S 1991 Identification of the Lamé coefficients from boundary observations Inverse Problems 7 335-54

[2] Astala K and Päivärinta L 2006 Calderón's inverse conductivity problem in the plane Ann. Math. 163 265-99

[3] Bukhgeim A 2008 Recovering the potential from Cauchy data in two dimensions J. Inverse Ill-Posed Problems 16 19-34

[4] Calderón A P 1980 On an inverse boundary value problem Seminar on Numerical Analysis and Its Applications to Continuum Physics (Río de Janeiro: Sociedade Brasileira de Matemática) pp 65-73

[5] Eskin G 2001 Global uniqueness in the inverse scattering problem for the Schrödinger operator with external Yang-Mills potentials Commun. Math. Phys. 222 503-31

[6] Eskin G and Ralston J 2002 On the inverse boundary value problem for linear isotropic elasticity Inverse Problems 18 907-21

[7] Ikehata M 1990 Inversion formulas for the linearized problem for an inverse boundary value problem in elastic prospection SIAM J. Appl. Math. 50 1635-44

[8] Ikehata M 1993 An inverse problem for the plate in the Love-Kirchhoff theory SIAM J. Appl. Math. 53 942-70

[9] Ikehata M 1996 A relationship between two Dirichlet to Neumann maps in anisotropic elastic plate theory J. Inverse Ill-Posed Problems 4 233-43

[10] Ikehata M, Nakamura G and Yamamoto M 1998 Uniqueness in inverse problems for the isotropic Lamé system J. Math. Sci. Univ. Tokyo 5 627-92

[11] Imanuvilov O, Uhlmann G and Yamamoto M 2010 The Calderón problem with partial data in two dimensions J. Am. Math. Soc. 23 655-91

[12] Imanuvilov O, Uhlmann G and Yamamoto M 2011 Determination of second-order elliptic operators in two dimensions from partial Cauchy data Proc. Natl Acad. Sci. USA 1008 467-72

[13] Imanuvilov $\mathrm{O}$ and Yamamoto M 2011 Reconstruction of the Lamé parameters from partial Cauchy data J. Inverse Ill-Posed Problems 19 881-91

[14] Nachman A 1996 Global uniqueness for a two-dimensional inverse boundary value problem Ann. Math. 143 71-96

[15] Nakamura G and Uhlmann G 1993 Identification of Lamé parameters by boundary measurements Am. J. Math. 115 1161-87

[16] Nakamura G and Uhlmann G 1994 Global uniqueness for an inverse boundary value problem arising in elasticity Invent. Math. 118 457-74

Nakamura G and Uhlmann G 2003 Invent. Math. 152 205-7 (erratum)

[17] Nakamura G and Uhlmann G 1995 Inverse boundary problems at the boundary for an elastic system SIAM J. Math. Anal. $26263-79$

[18] Nakamura G and Uhlmann G 2002 Complex geometrical optics solutions and pseudoanalytic matrices Ill-posed and Inverse Problems ed S I Kabanikhin and V G Romanov (Utrecht: VSP) pp 305-38

[19] Sylvester J and Uhlmann G 1986 A uniqueness theorem for an inverse boundary value problem in electrical prospection Commun. Pure Appl. Math. 39 91-112

[20] Uhlmann G 2009 Calderón's problem and electrical impedance tomography Inverse Problems 25123011 